\title{
Personality and Emotion Regulation Strategies
}

\author{
Personalidad y estrategias de regulación emocional \\ Esti Hayu Purnamaningsih ${ }^{1 *}$
}

\begin{abstract}
The emotions has many important functions in our life such as in relation of interpersonal communication, and health. In interpersonal communicative function aimed to signal to other information about internal state. Emotions manifests in specific cognitive, behavioural, and physiological reactions, thus closely related to health. There is wide variety of ways for individuals to regulate their emotion. In this regard, there are two kinds of emotion regulation strategy; first Antecedent-focused emotion regulation consisting of situation selection, situation modification, attentional deployment, cognitive change and second, Response-focused emotion regulation consisting of suppression. The purpose of this research is to investigate personality factors relate with emotion regulation strategies. 339 students from Faculty of Psychology, Universitas Gadjah Mada were participating in this study and given The Big Five Personality Factors (Ramdhani, 2012, adaptation), and the modified version of the Emotion Regulation Scale was used, Emotion Regulation Questionnaire (John \& Gross, 2004) which measure personality and emotion regulation respectively. Using multiple regression analysis, the study indicated that personality predicts emotion regulation strategies.
\end{abstract}

\section{Resumen}

Las emociones tienen muchas funciones importantes en nuestra vida, como en relación de comunicación interpersonal y salud. En la función comunicativa interpersonal se pretende señalar a otros, información sobre estados internos. Las emociones se manifiestan en reacciones cognitivas, conductuales y fisiológicas específicas, por tanto estrechamente relacionadas con la salud. Hay una gran variedad de formas para que los individuos regulen su emoción; en este sentido, hay dos tipos de estrategia de regulación emocional. La primera, regulación de la emoción centrada en los antecedentes: consistente en la selección de la situación, la modificación de la situación, el despliegue atencional y el cambio cognitivo. La segunda, la regulación de la emoción centrada en la respuesta consistente únicamente en la supresión. El propósito de esta investigación es investigar los factores de personalidad que se relacionan con las estrategias de regulación emocional. 339 estudiantes de la Facultad de Psicología de la Universidad de Gadjah Mada participaron en este estudio y se les administró la escala de los cinco gran factores de personalidad (adaptación de Ramdhani, 2012) y la versión modificada de la escala de regulación emocional, el cuestionario de regulación emocional (John \& Gross, 2004) que miden la regulación de la personalidad y la emoción respectivamente. El estudio indicó que la personalidad predice las estrategias de regulación de la emoción usando el análisis de regresión múltiple.

\section{Keywords}

Personality; emotion regulation; strategies.

Palabras Clave

Personalidad; emoción; estrategias de regulación.

${ }^{1}$ Faculty of Psychology Universitas Gadjah Mada, Indonesia.

*Corresponding author: esti@ugm.ac.id

Manuscript received 01-01-2016; Revised 03-07-2016; Accepted 18-12-2016.

\section{Introduction}

Emotion is very important in everyday life, because emotional expression helps to provide information about the status of individual interaction with others and with the environment
(Gross \& John, 2003). Emotions have usually been studied with regard to three components: cognitive-experiential ("feelings"), behavioural-expressive (e.g. facial expression), and physiological-biochemical (physical states). The concept of 
emotion regulation refers to the process of altering stimuli and modulating responses which consist of those three components (Eisenberg, 2004; Thompson, 1994). For instance certain expression such as sad, angry, unhappy, ashamed or afraid, panic, could help others in determining their response. This is in accordance with Frijda (in Denollet, Nyklicek, \& Vingerhoets, 2008), who suggest that besides aiding interpersonal communication, emotions are in fact also closely related to physiological reaction. Emotional responses have indeed been linked to nervous system and certain physiological changes in the body (Strongman, 2003). Emotional response will induce physiological changes in the body, such as hot face, palpitations (Passer \& Smith, 2008). In particular, anger can increase the blood pressure, heart rate and create muscle tension (Denollet et al., 2008). Chen (2016) was found that cognitive assessment could reduce the emotional experience, reduce the activation of the physiological response and sympathetic nervous system, while the inhibition of expression was able to reduce emotional behaviour, but the physiological response and sympathetic nervous system activation was enhanced. That was supported by Nezamipour and Ahadi (2016), emotion regulation and management play a determinant role in health and prevention of mental disorders in patients with chronic disease.

The information indicates that emotion plays a significant role in maintaining health. Therefore, in everyday life emotions need to be managed properly. Emotion regulation is.a cognitive reappraisal strategy to forward aversive emotional reduce the negative and social consequences (Gross, 1998; Matsumoto, Nakagawa, \& Sanae, 2008; Volokov, 2008). Liliana and Nicoleta (2014) founded that emotional stability predicts wellbeing on all four dimensions: positive affects, negative affects, emotional distress and life satisfaction. Emotion regulation strategies are predictors for (positive and negative) affects only, and not for emotional distress or life satisfaction.

People regulate their emotions in different ways. A strategy chosen for regulating emotion can be more healthier than others (John \& Gross, 2004), or more effective than others (Lopes, Salovey, Coté, \& Beers, 2005). Gross and John (2003) showed, that reappraisal is positively related to well-being, in contrast another technique,called emotional suppression is negatively correlated with well-being (Matsumoto, 2006) Suggests that there might be individual differences in emotion regulation in a different culture, it is possible for their individual differences in emotion regulation due to differences in culture: this could be manifested further in personality traits. Bolger and Zuckerman (1995) demonstrated that those with high neuroticism tended to use ineffective emotional regulation strategy than those who scored less in a questionnaire aimed to measure neuroticism susceptibility in individuals (Dynes, 2010).

Based on the above explanation, there appears a question; how individual's personality traits relate with emotion regulation strategies? Therefore, this research aims to find out whether there is a relationship between personality factors and emotion regulation strategies.

\subsection{Emotion regulation strategies}

Gross (2007) differentiates between antecedent-focused strategies and response-focused strategies in regards to emotional regulation. Antecedent-focused strategies deal with what is about to happen in regards with emotional response, such as the occurrences prior to emotional events. On the other hand, Response-focused strategies deal with emotional events when it is on progress. There are four different type of Antecedentfocused strategies: 1) situation selection, 2) situation modification, 3) attentional deployment, 4) cognitive change. The other strategy a response modulation, is signature type of a response-focused strategy.

In the situation selection strategy, one can approaches or avoids certain people or situations based on what potential emotional events may be resulted from the interaction. One option to be choosing a situation that suits their emotional response level (Gross, 1998). For example, one may choose to stay at home rather than going to a party because they have a conflict with someone whom they believe is also going to the party.

Emotion regulation strategy on the other hand through situation modification in which one modifies an environment to alter its emotional impact For example, when watching a bothersome television program, one may shift to other channel.Further emotion regulation strategy can work through attentional deployment, in which one turns attention toward or away from something to preserve their emotions. For example, one may close their eyes when witnessing an execution with gunshot. In addition, emotion regulation strategy can be undergone through cognitive change in which one makes decide a cognitive meaning of an incident. A typical technique of cognitive change strategy, is reappraisal. Reappraisal takes a form as a cognitive manipulation that involves construing a potentially emotion eliciting situation in away that changes its emotional impact. Lastly, emotion regulation strategy through response modulation is a type strategy which focuses on response when the emotional events in under way. It is applied after any liable emotion response has been initiated. One specific technique of response modulation is suppression, in which inhibits an ongoing expression of emotion. John and Srivastava (in John \& Gross, 2004) stated that there is differences in emotion regulation of adults caused by the variation of their personality dimensions.

\subsection{Personality}

Personality is an important aspect in understanding human beings. Feist and Feist (2006) defined personality as a pattern that relatively stable, consisting of unique traits, dispositions or characteristics within an individual that show some consistent measures about his/her behaviour. Allport (in Feist $\&$ Feist, 2006) stated that personality is a dynamic organization within an individual, which constitutes a psychophysical system. It determines the individual's self-adjustment with 
environment. It can be concluded that personality is a set of characteristics that relatively settles within an individual for self-adjusting with environment.

There are some approaches to understand theory of personality such as Psychodynamic Approach, Humanistic Approach, Traits Approach, Learning Theory Approach, Cognitive Approach and Socio-cognitive Approach (Pervin \& John, 2001). Psychodynamic Approach views personality as a result of interplaying dynamics of motives, drives, needs and conflicts within an individual. In addition, to that, it also emphasizes the importance of consciousness aspect. Those who practice humanistic approach believe that every human being essentially has positive potentials that can be developed. It also emphasizes individual's subjective experience and relates to cognitive perception and process.

The traits approach, on the other hand, put emphasis on the relatively stable characteristics of personality in different situations. There is a specific dimension inherent within an individual since birth. The approach of learning theory in contrast, rejection-adaptability, and unchangeable structure. The view personality as a matter that can change; as well as behaviours which can be learned. Almost relevant, the Socio-cognitive approach puts emphasis on interplay between human thought and social environment that enables learning process.

The study used the Traits Approach because it emphasizes on the characteristics of personality that is stable even in different situation. Traits are constant patterns of an individual's behaviour, feeling and thinking. Most researchers with this approach use procedure of factor analysis statistics to determine basic traits existing in an individual's personality (Pervin \& John, 2001).

According to Feist and Feist (2006), study on traits was firstly initiated by Allport and Odbert in the 1930s, continued by Cattell in the 1940s and then in the 1960s by Tupes, Christal and Norman. In 1963, Norman (in Pervin \& John, 2001) did a research using factor analysis, and found five basic factors of personality. According to Five Factor Model (FFM) of traits, personality is described in the form of five major dimensions (McCrae \& Costa J. in Pervin \& John, 2001). These five basic dimensions are Openness to Experience, Conscientiousness, Extraversion, Agreeableness, and Neuroticism. Further Howard (Pervin \& John, 2001) argued that Openness to experience is characterized by discretion, profoundness and mental complexity. While Conscientiousness refers focus their attention to goals and efforts to achieve the goals. They are career-oriented. In addition, they are flexible, more impulsive, convincing in doing their jobs. Extraversion and agreeableness on the other hand, focus on individual's relationship with others. An extroverted individual has tendency to be active, both physically and verbally, while an introvert one tends to be dependent and aloof. Agreeableness refers to tendency to conform with social interest. A person with trait is caring, supportive, sympathetic and warm. They are not self-centered persons. Neuroticism is the opposite from emotion stability in identifying negative emotion, including anxiety, sadness and tension.

\subsection{Personality of Emotion Regulation Strategy}

According to Allport (Gross, 2007), traits of personality make individuals having disposition of responses that consistency lead to adaptive and behaviors. Differences among individuals in the strategies of emotion regulation represent the traits of their personality. John and Srivastava (in Gross, 2007) suggested that Big Five is among few personality structure that has an integrative function. Its construction manifests in groups of behavioural, emotional and cognitive responses. Further, John and Srivastava (Gross, 2007) explained about the relationship between each personality trait and strategy of emotion regulation as follows:

Openness to experience. Individuals with this trait have wide-ranging, deep cognitive contents and authentic as well as complex life experiences. Their openness to others' emotional situations in general enables them to be optimistic individuals and enable them in the emotion regulation strategy, i.e. cognitive change. In addition, people with this trait are also are good at using the strategies of situation selection and situation modification, depending on any situation they face.

Conscientiousness. socially determines control of impulse that mediates goal-accomplishing behaviours such as thinking before doing, cancelling happiness, following the norms, planning, and managing and prioritizing jobs. These behaviours enable them to do emotion regulation strategy i.e. situation selection. Individual with high conscientiousness also often modifies situation; to reduce the aversive consequence of emotions events, In addition, they tend to do the emotion regulation strategy i.e.attentional deployment to accomplish their goals. Focusing on job and deploying attention to relevant goal is one of the characteristics of conscientiousness.

According to John and Srivastava (in Gross, 2007), individuals with extraversion trait, tend to show enthusiasm for social life; they are assertive, gregarious and energetic. They tend to always have positive emotions, yet not reluctant to express the negative ones. They are very careful in selecting situation, opting more to approach situations that are potentially beneficial for them or those who potentially generating positive emotions. In contrast, introvert individuals tend to avoid or withdraw from many situations.

Agreeableness on the other hand refers to interpersonal relationship in the future. It is opposite from traits like disbelief in others, antagonism. Unlike other traits, agreeableness can not be linked to specific emotion regulation strategy because the effect of agreeableness depends on specific relationship. Individuals with high score of agreeableness tend to pay attention to others' interest more that their own interests. Consequently, they do not try hard to change situation in order to preserve emotional state.

Neuroticism is opposite to stability of emotion. It involves certain emotions like anxiety, worry, sadness and tension. In general it has negative relationship with the strategy of emo- 
tion regulation. Neurotic individuals hardly believe that others can change their emotions and in fact their emotions are hard to control (Gross, 1998b). They tend to be pessimistic in making any strategy for regulating their emotions because they are afraid of facing failure. In short, Gross (2007) presented unique relationship between personality factors and emotion regulation strategies. in studies of personality related to emotional experiences or emotion expression, neuroticism usually is linked with negative emotional experience (Schimmack et al. in Matsumoto, 2006). The other trait, such as extraversion has correlation also with both positive and negative emotions. Aspects of extraversion such as warmness, assertiveness, vigor, positive emotions have relationship with emotion regulation (McCrae \& Costa, in Livingstone \& Srivastava, 2014; Matsumoto, 2006).

According to Matsumoto (2006), there is possible relationship between other personality traits and emotion regulation; for instance, between neuroticism and negative expression, agreeableness and positive emotion, conscientiousness and positive emotion. In addition, Matsumoto et al. (2008) found a relationship between openness and the ability to recognize emotion. John and Gross (2004) stated that there is differences in neuroticism and extraversion within the development of the use of reappraisal and suppression strategies. It is supported by the study done by Wang, Shi, and Li (2009) which found that there is relationship between extraversion, neuroticism and positive and negative emotions, mediated by the contribution of reappraisal and suppression.

Based on the existing theories and previous research, the hypothesis of this research was: Personality is predictor for emotion regulation strategies.

\section{Method}

\subsection{Subjects}

The subjects consisted of 339 second and third Psychology students at Universitas Gadjah Mada. The sample consists of 75 males and 264 females. Subjects aged between $17-19$ years. Subjects voluntarily participated in this study. They do not compensated financially, but receive a ballpoint and snacks upon the task completion. Subsequently after the task, the students were given a lecture on how to manage emotions in everyday life.

\subsection{Variables of the Research}

The independent variables of this study is personality traits included in the Big Five Theory, namely Openness to experience (O), Conscientiousness(C), Extraversion (E), Agreeableness (A), and Neuroticism (N). The dependent Variables were two strategies of emotion regulation, i.e. Antecedent focused emotion regulation, consisting Situation Selection (SS), Situation modification (SM), Attentional Deployment (AD) and Cognitive Change (CC) or Reappraisal, and the Response focused emotion regulation, consisting Suppression.

\subsection{Operational Definitions}

Personality is a thinking pattern and way of an individual perceives his/her traits consistently. Personality in this research was measured with the Big Five Personality Scale made by John and Srivastava (Pervin \& John, 2001), which was adapted by (Ramdhani, 2012) consisting of five traits of personality, namely Openness to experience, conscientiousness, Extraversion, Agreeableness, and neuroticism.

Openness to experience operationalized as being eager to get more ideas open to new ideas, attentive to both positive and negative emotions. Conscientiousness is defined as hardworking, self-discipline, punctual and accurate. Extraversion is of being open, optimistic, gregarious, warm, energetic and interested in social activities. Agreeableness is of being easy to be influenced, cooperative, sympathetic, warm and oriented to others' interest. Lastly, Neuroticism operationalized as being easy to be anxious, temperamental and moody.

Emotion Regulation Strategy is a strategy or a way used by individuals to manage and express their emotions and feelings in their daily lives. There are two groups of strategy that were measured, namely Antecedent focused emotion regulation, consisting Situation Selection, in which an individual chooses to be in any situation suitable for them; Situation Modification, in which an individual changes a situation to get different scale of emotion; Attentional Deployment which refers to shifting of attention to certain aspect; Cognitive Change / Reappraisal, which refers to acts of changing one's cognitive meaning to reduce emotion, and Response focused emotion regulation, consisting of suppression, a way of managing response by obstructing any on-going behavioural expression.

\subsection{Instruments}

a) Big Five Personality Factor (adapted by Ramdhani, 2012). The scale of personality consists of 5 traits, namely Openness to experience, Conscientiousness, Extraversion, Agreeableness, and neuroticism. The number of item was 44 . The item scores moved from not very suitable (1), "not suitable" (2), "rather suitable" (3), "suitable" (4) and "very suitable" (5) for both favorable and unfavorable items. Ramdhani (2012) adapted the language and culture of The Big Five Inventory (BFI), through the process of translation, from the English into Indonesian by 2 Indonesian whose background is in psychology Results were translate into English by a translator whose background psychology, then the results were compared with the original scale to check the congruency of meaning.

Next the scale was read by three Indonesia people, to determine whether the language can be understood, and then given to 790 people with various backgrounds. Confirmatory factor analysis (CFA) propose two measuring models, namely M2 and M3. The M2 model consisted of 37 items whose quality was good enough, showing the score of $\lambda$ ranging between 0.32 and 0.78 and the reliability $\alpha$ between 0.70 and 0.79 . The M3 models consisted of 28 items with good quality, showing the score of $\lambda$ between 0.43 and 0.80 and its reliability $\alpha$ between 0.70 and 0.79 . 
b) Emotion Regulation Scale is a modification of Emotion Regulation Questionnaire (ERQ) made by Gross (1998) which mean to identify cognitive change or reappraisal strategy and suppression strategy. Gross (2007) suggested, that for measuring the antecedent focused strategies, recommended not only cognitive change or reappraisal but also situation selection, situation modification, attentional deployment and suppression. Subsequently researcher made 50 items in accordance with the concept of each factor. The items were made in Indonesian language. The item scores ranging from "not very suitable" (1), "not suitable" (2), "rather suitable" (3), "suitable" (4) and "very suitable" (5), applicable otherwise for unfavorable items. The total scores obtained indicate the extent to which individuals use the emotion regulation strategies. After testing, we found 12 items which has low different power, resulting 38 valid items remaining in the scale.

Before being applied, scale was tested to know its reliability. The results are, strategy selection situation has alpha reliability index $\alpha=0.685$; situation modification has alpha reliability $\alpha=0.712$, attentional deployment has alpha reliability index $\alpha=0.571$; cognitive change has alpha reliability index $\alpha=0.716$ and suppression have alpha reliability $\alpha=0.780$.

\subsection{Procedure of the Research}

Data collecting was done at the Faculty of Psychology, Universitas Gadjah Mada using two scales, namely The Big Five Personality Scale and Emotion Regulation scale. Out of 346 copies of the scales having been filled, we could only analyse 339 could be analysed because the other 7 were incomplete. The data were analysed using multiple regression analysis statistics with stepwise method. In this case, regression analysis was conducted 5 times due to 5 traits of personality in which each was correlated with every type of emotion regulation strategy. The computation was carried out using SPSS program. The next step was to find out how effective contribution of each personality trait to each emotion regulation strategies.

\section{Results}

Multiple regression analysis was used to test whether personality traits significantly predicted emotion regulation. The analysis was conducted separately for each emotion regulation strategies so that there are five regression analyses. For selection situation, the result of the regression analysis indicated that of among five personality factors, only neuroticism significantly predicts selection situation $\left(R^{2}=.097, F=36.250\right.$, $p<0.01)$. However, openness $(\beta=.159, p<.01)$, extraversion, $(\beta=.272, p<0.01)$ and agreeableness $(\beta=.195$, $p<0.01)$ significantly predict situation modification emotion regulation strategy. The simultaneous analysis found that those variables explain $20.3 \%$ variance of outcome variables $\left(R^{2}=.203, F=28.415, p<0.01\right)$.

Three variables explained $8.3 \%$ of the variance attentional deployment emotion regulation strategy $\left(R^{2}=.017\right.$,
$F=10,043, p<0.01)$. Those variables and their estimated value are extraversion $(\beta=.152, p<.05)$, agreeableness $(\beta=.145, p<0.05)$ and Neuroticism accounted for the most variance $(\beta=.249, p<.01)$. For cognitive change emotion regulation strategy, three predictors explained $25.8 \%$ of the variance $\left(R^{2}=.258, F=38.733, p<0,01\right)$. Those variables are Conscientiousness $(\beta=.161, p<.01)$, Extraversion $(\beta=.146, p<0.01)$ and Agreeableness that accounted most variance $(\beta=.348, p<.01)$. For Suppression emotion regulation strategy, three predictors explained $24.9 \%$ of the variance $\left(R^{2}=.249, F=37.036, p<0.01\right)$. It was found that Agreeableness significantly predicted Suppression Emotion regulation strategy $(\beta=.118, p<.05)$ as Neuroticism $(\beta=-.279, p<0.01)$ and Extraversion accounted for the most variance $(\beta=-.562, p<.01)$.

\section{Discussion}

According to Allport (Gross, 2007), traits of personality make an individual having disposition of responses that lead to doing and expressive behaviours. Differences among individuals in the strategies of emotion regulation may root from the traits of their personality.

John and Srivastava (Gross, 2007, in) explained that one of structures of personality that have integrative function is the Big Five. Its construction manifests in groups of behavioural, emotional and cognitive responses. John and Srivastava (Gross, 2007) explained about the relationship between each personality trait and strategy of emotion regulation.

The Result indicated that Openness, together with Agreeablenes, and Extraversion support the use situation modification strategy. In this study, Openness acted as a predictor of situation modification. Openness has deep cognitive contents, authentic and complex life experiences. Their openness trait to others' emotional situations in general enables them to be optimistic individuals. They also are good in at using the strategies of situation modification, depending on any situation they face. Those who are susceptible to Oppeness trait would prefer. In other words those people preferentially interact with specific situations (Gross, 2007), thus in certain situations individuals are encourage to make modifications.

Conscientiousness, together with Extraversion, and Agreeableness support the use of cognitive change emotional regulation strategy. Conscientiousness socially determines control of impulse that mediates goal-accomplishing behaviours such as thinking before doing, cancelling happiness, following the norms, planning, and managing and prioritizing jobs. Individual with high conscientiousness will change his mind, to reduce negative emotion. Javaras et al. (2012) found that conscientiousness predicted recovery from negative emotion; In addition, Balliet (2010) also found there was a positive relationship between conscientiousness and forgiveness. Sorić, Penezić, and Burić (2013) found that the series of multiple hierarchical regression analyses showed that all groups of predictors have made significant and independent contribution to the explanation of all analysed emotions. Conscientious- 
Personality and Emotion Regulation (Research Article) - 58/60

Table 1. Correlation Matrix of Variables used in this study

\begin{tabular}{|c|c|c|c|c|c|}
\hline Personality & SS & SM & $\mathrm{AD}$ & $\begin{array}{l}\mathrm{CC} / \\
\text { Reappraisal }\end{array}$ & $\begin{array}{l}\mathrm{RM} / \\
\text { Suppression }\end{array}$ \\
\hline Openness & .011 & $.241 * *$ & .046 & $.173 * *$ & -.038 \\
\hline Conscientiousness & $-.125^{*}$ & $.257 * *$ & .004 & $.319 * *$ & $-.177 * *$ \\
\hline Extraversion & $-.098^{*}$ & $.384 * *$ & $.111^{*}$ & $.337 * *$ & $-.412 * *$ \\
\hline Agreeableness & -.065 & $.309 * *$ & $.163 * *$ & $.450 * *$ & -.048 \\
\hline Neuroticism & $.312 * *$ & . - -.087 & $.169 * *$ & $-.224 * *$ & -.084 \\
\hline
\end{tabular}

Note. Information: $*=p<0.05 * *=p<0.01$.

ness and Agreeableness lost their predictive significance for experiences of unhappiness and anger.

Extraversion is one of four personality traits that contributes the most to situation modification strategy. Persons with high enthusiasm for social life shows unique characteristics such as assertive, gregarious and energetic. They tend to always have positive emotions, modify situation to feel better emotions. Verduyn and Brans (2012) used correlational ana-lyses and revealed that extraversion was positively related to frequency, intensity and duration of positive emotions whereas neuroticism was positively related to the frequency and duration of negative emotions. Regression analyses and a comparison of effect sizes further showed that the duration of positive emotions was the strongest predictor of extraversion whereas the frequency of negative emotions is the strongest predictor of neuroticism. It was supported by research from Nezamipour and Ahadi (2016) that personality traits play an important role in emotion regulation strategies as well as quality of life in dialysis patients. Other researchers, Wang et al. (2009) founded that individuals with different personality dimensions (i.e., extraversion and neuroticism) use emotion regulation strategies differently (i.e., reappraisal), which in turn influences the affect they experience. Research by Vantieghem, Marcoen, Mairesse, and Vandekerckhove (2016) indicated that in the evaluation of subjective sleep, the impact of personality and emotion regulation processes, such as emotion suppression, should be taken into account.

In addition to that extraversion also became a predictor for attentional deployment and cognitive change. Through attentional deployment strategy, some one turns attention toward or away from something in order to influence their emotions, while Extraversion are assertive, and having freedom to express negative ones. Cognitive change is a way of deciding a cognitive meaning of an incident, a special kind of cognitive change, which aims to reduce emotion is reappraisal - Reappraisal is a form of cognitive change that involves construing a potentially emotion eliciting situation in away that changes its emotional impact. People with extraversion trait shows characteristics such as having high enthusiasm for social life; assertive, gregarious and energetic. They tend to always have positive emotions, therefore they will maintain their positive emotion. In this study it was found Extraversion be a negative predictor for suppression strategies, that people who have high extraversion tend to use energy, social skills, and trying to express emotions such as by modify the situation and cognitive change (Gross, 2007, Carver \& White, in). Tackman and Srivastava (2016) explained that personality judgements and other impressions of targets who either suppressed or expressed their emotion expressive behaviour to amusing or sad film clips. In replicated research, subjects who suppressed either amusement or sadness were judged as less extraverted, less agreeable, and more interpersonally avoidant and anxious than subjects who expressed, and participants were less interested in affiliating with suppressors compared to expressers.

Agreeableness refers to tendency for prioritizing social interest. A person with this trait shows characteristics such as is caring, supportive, and warm. Kuppens (2005) found, both agreeableness and perceived social esteem were negatively related to trait anger. In this study, agreeableness contributed toward situation modification strategy, Interestingly, our finding is contradictory from Gross's statement (Gross, 2007), as he assumed that people with this trait did not try hard to change situation while maintaining emotion. It is possible that what was done in order to maintain relationships with others has comforted her. Agreeableness also contributes for other emotion regulation strategies, such as Attentional Deployment, Cognitive Change, even for Suppression. As proposed by Gross (2007), people with Agreeableness do not try hard to change situation, while preserving their emotion. Instead, people with high degree of Agreeableness were gentle, and depends on specific relationship in the future, so they use Attentional deployment strategy. In the strategy, one's turn attention toward or away something in order to influence one's emotions. The use of suppression strategy in order to maintain relationships with other people, because people with high degree of agreeableness were tend to pay attention to others' interest more that their own interests. Based on the characteristics of that, agreeableness perform cognitive change.

The Result indicated that situation selection is most used by neuroticism. Neuroticism tend to be pessimistic in making any strategy for preserving regulating their emotion, because they are afraid of going through failure (Gross, 1998b). Neuroticism is opposite to stability of emotion. It involves emotions like anxiety, worry, sadness and tension. Liliana and Nicoleta (2014) showed that emotional stability predicted well-being on all four dimensions: positive affects, negative 
affects, emotional distress and life satisfaction. Emotion regulation strategies are predictors for (positive and negative) affects only, and not for emotional distress or life satisfaction. Neuroticism will choose a situation which they feel safe, and don't use more risky, situation therefore they choose situation selection strategies when facing emotional events. Besides that, neurotic people tended to use Attentional deployment emotion regulation strategy. The strategy is an easy way to deal with anxiety, fears, grief and pressure of the shifting center of attention. (Dynes, 2010) found that Neuroticism reported maladaptive strategies. People with high Neuroticism were choosing maladaptive strategies, it can be inferred that the maladaptive strategy use is being maintained by the reduction of negative affect.

\section{Limitations}

In the end, it is necessary to highlight warn that this study has certain limitations. first, this study was conducted on a sample of college students, which, to some extent, limits the possibility of generalization of its conclusions. Second, his study examined the emotions that students usually experience in everyday life's scenario. For this reason, the students were requested to recall how they typically feel in everyday life. This recall processes may lead to some kind of distortions and diminishing intensity of extreme emotions, which reduces their variability. The same problem was also present when measuring emotional regulation strategies. Associated with these, potential problems that occur when using self-report methods - such as giving socially desirable answers. In future research it should be interesting and important to test the observed relations in different methods. In this sense, experimental approaches are required in order to gain a deeper understanding of the dynamics of the emotion generative process.

\section{Conclusion}

The present research has mainly confirmed theoretical predictions that personality predicts emotion regulation strategies. Among the five personality factors only neuroticism significantly predicts selection situation strategy. However, openness, extraversion, and agreeableness significantly predict situation modification emotion regulation strategy. Extraversion accounted for the most variance.

Attentional deployment emotion regulation strategy was more correlated with extraversion, agreeableness and neuroticism. Neuroticism accounted for the most variance. For cognitive change emotion regulation strategy, three predictors explained.Those variables are Conscientiousness, Extraversion and Agreeableness that accounted most variance. Further it was found that Agreeableness, Neuroticism, and Extraversion significantly predicted Suppression Emotion regulation strategy, in which Extraversion accounted for the most variance.

\section{References}

Balliet, D. (2010). Conscientiousnes and forgiveness: A metaanalysis [Journal Article]. Personality and Individual Differences, 48, 259-263.

Bolger, N., \& Zuckerman, A. (1995). A framework for studying personality in the stress process [Journal Article]. Journal of Personality and Social Psychology, 69(5), 890-902.

Chen, H. (2016). A theoretic review of emotion regulation [Journal Article]. Open Journal of Social Sciences, 4, 147-153.

Denollet, J., Nyklicek, I., \& Vingerhoets, J. M. (2008). Emotion regulation [Book]. Springer.

Dynes, M. (2010). Neuroticism and emotion regulation (Thesis).

Eisenberg, N. (2004). Emotion-related regulation: An emerging construct [Journal Article]. Merrill-Palmer Quarterly, 50, 236-259.

Feist, J., \& Feist, G. J. (2006). Theories of personality (6th ed.) [Book]. Boston, Mass: McGraw-Hill.

Gross. (1998). Antecedent-and response-focused emotion regulation: divergent consequences for experience, expression, and physiology [Journal Article]. Journal of Personality and Social Psychology, 74(1), 224-237.

Gross. (1998b). The emerging field of emotion regulation: An integrative review [Journal Article]. Review of General Psychology, 7(3), 271-237.

Gross. (2007). Handbook of emotion regulation [Book]. New York: The Guilford Press.

Gross, \& John, O. P. (2003). Individual differences in two emotion regulation processes: implications for affect, relationships, and well-being [Journal Article]. Journal of Personality and Social Psychology, 85(2), 348-362.

Javaras, K. N., Schaefer, S. M., van Reekum, C. M., Lapate, R. C., Greischar, L. L., Bachhuber, D. R., ... Davidson, R. J. (2012). Conscientiousness predicts greater recovery from negative emotion. [Journal Article]. Emotion, 12(5), 875-81. doi: 10.1037/a0028105 .Epub2012May28.

John, O. P., \& Gross, J. (2004). Healthy and unhealthy emotion regulation: Personality processes, individual differences, and life span development [Journal Article]. Journal of Personality \& Social Psychology, 72(6).

Kuppens, P. (2005). Interpersonal determinants of trait anger: low agreeableness, perceived low social esteem, and the amplifying role of the importance attached to social relationships [Journal Article]. Personality and Individual Differences, 38, 13-23.

Liliana, B., \& Nicoleta, T. M. (2014). Personality, family correlates and emotion regulation as wellbeing predictors [Journal Article]. Social and Behavioral Sciences, 159, 142-146.

Livingstone, K. M., \& Srivastava, S. (2014). Personality and positive emotion [Book Section]. In . L. K. In M. Tugade M. Shiota (Ed.), Handbook of positive emotions. 
New York: Guilford.

Lopes, P. N., Salovey, P., Coté, S., \& Beers, M. (2005). Emotion regulation abilities and the quality of social interaction [Journal Article]. Emotion, 5(1), 113-8.

Matsumoto, D. (2006). Are culture differences in emotion regulation mediated by personality traits? [Journal Article]. Journal of Cross-Cultural Psychology, 37(4), 421-437.

Matsumoto, D., Nakagawa, S., \& Sanae, H. Y. (2008). Culture, emotion regulation, and adjustment [Journal Article]. Journal of Personality and Social Psychoogy, 94(6), 925-937.

Nezamipour, E., \& Ahadi, H. (2016). The effect of cognitive emotion regulation strategies on the relationship between personality traits and quality of life in dialysis patients [Journal Article]. Qom University of Medical Sciences Journal, 10(2), 70-81.

Passer, M. W., \& Smith, R. E. (2008). Psychology: the science of mind and behavior [Book]. New York: McGrawHill.

Pervin, L. A., \& John, O. P. (2001). Personality: Theory and research [Book]. New York: Wiley.

Ramdhani, N. (2012). Adaptasi bahasa dan budaya inventori big five [Journal Article]. Jurnal Psikologi, 39(2), 189207.

Sorić, I., Penezić, Z., \& Burić, I. (2013). Big five personality traits, cognitive appraisals and emotion regulation strategies as predictors of achievement emotions [Journal Article]. Psychological Topics, 22(2), 325-349.

Strongman, K. T. (2003). The psychology of emotion, from everyday life to theory (5th ed.) [Book]. England: John Wiley \& Sons Ltd.

Tackman, A. M., \& Srivastava, S. (2016). Social responses to expressive suppression: The role of personality judgments [Journal Article]. Journal of Personality and Social Psychology, 110, 574-591.

Thompson, R. A. (1994). Emotion regulation: A theme in search of definition [Book Section]. In I. N. A. Fox (Ed.), The development of emotion regulation: Biological and behavioural considerations (p. 25-52). Chicago: University of Chicago Press.

Vantieghem, I., Marcoen, N., Mairesse, O., \& Vandekerckhove, M. (2016). Emotion regulation mediates the relationship between personality and sleep quality [Journal Article]. Psychology \& Health, 31(4), 1-18.

Verduyn, P., \& Brans, K. (2012). The relationship between extraversion, neuroticism and aspects of trait affect [Journal Article]. Personality and Individual Differences, 52, 664-669.

Volokov, R. N. (2008). Cognitive and physiological correlates of emotion regulation: Is reappraisal a teachable skill? (Thesis).

Wang, L., Shi, X., \& Li, H. (2009). Neuroticism, extraversion, emotion regulation, negative affect and positive affect: the mediating roles of reappraisal and suppres- sion [Journal Article]. Social Behavior and Personality, 37(2), 193-194. 